\title{
Using quantitative imaging to determine the correlation between hypothalamic inflammation and anxiety and depression in young patients with obesity
}

\author{
Guan-Zhong Dong^, Hao-Jun Yang, Yu-Wen Jiao, Qiao-Yang Zhang, Yi Ma, Shu-Min Zhu, \\ Li-Hao Zhang, Min Zhang, Tian Yao, Yin Cao, Li-Ming Tang \\ Department of Neurology, Changzhou No.2 People's Hospital Affiliated to Nanjing Medical University, Changzhou, China \\ Contributions: (I) Conception and design: QY Zhang, SM Zhu; (II) Administrative support: Y Cao, LM Tang; (III) Provision of study materials or \\ patients: HJ Yang, YW Jiao; (IV) Collection and assembly of data: Y Ma, LH Zhang; (V) Data analysis and interpretation: GZ Dong, M Zhang, T \\ Yao; (VI) Manuscript writing: All authors; (VII) Final approval of manuscript: All authors. \\ Correspondence to: Yin Cao; Liming Tang. Department of Neurology, Changzhou No.2 People's Hospital Affiliated to Nanjing Medical University, \\ No.29, Xinglong Lane, Tianning District, Changzhou 213004, China. Email: 505376380@qq.com; drtangliming@163.com.
}

\begin{abstract}
Background: To investigate the incidence of anxiety and depressive disorders in young adults with obesity and the correlation between the severity of these disorders and hypothalamic inflammation.

Methods: The severity of anxiety and depressive disorders was assessed using the Self-Rating Anxiety Scale (SAS) and Self-Rating Depression Scale (SDS), respectively. Hypothalamic inflammation was evaluated by measuring the hypothalamus/amygdala (H/A) signal intensity (SI) ratio in T2-weighted phase quantitative magnetic resonance imaging (MRI).

Results: The incidence of depressive disorders in young (18-45 years) patients with obesity ( $\mathrm{n}=66)$ was higher than that in the control group $(n=44)$; anxiety disorder incidence did not differ significantly between groups. The bilateral H/A SI ratio in the obesity group was significantly higher than that in the control group. In the obesity group, there was no significant correlation between bilateral H/A SI ratio and body mass index (BMI) (right: $\mathrm{r}=-0.145, \mathrm{P}=0.721$; left: $\mathrm{r}=0.102, \mathrm{P}=0.415$ ) or SAS scores (right: $\mathrm{r}=-0.118, \mathrm{P}=0.444$; left: $\mathrm{r}=-0.295, \mathrm{P}=0.052)$; SDS scores were significantly correlated with left $\mathrm{H} / \mathrm{A}$ SI ratio $(\mathrm{r}=-0.353, \mathrm{P}=0.019)$, but not right $\mathrm{H} / \mathrm{A}$ SI ratio $(\mathrm{r}=-0.031, \mathrm{P}=0.843)$.
\end{abstract}

Conclusions: Patients with obesity had a higher incidence of depressive disorders. Left hypothalamus inflammation may be one of the links between obesity and depressive disorders.

Koywords: Anxiety; depression; hypothalamic inflammation; obesity

Submitted May 21, 2021. Accepted for publication Jul 02, 2021.

doi: 10.21037/apm-21-1480

View this article at: https://dx.doi.org/10.21037/apm-21-1480

\section{Introduction}

More than two-thirds of adults in the United States are overweight or obese (1), and there has been no significant decrease in this proportion in recent years (2). In China, approximately one-fifth of adults are overweight or obese (3); moreover, due to the large population base, the number of individuals with obesity in China is far greater than that in other countries. In addition to causing various cardiovascular and cerebrovascular diseases, obesity is also believed to be associated with mood disorders. Many studies have investigated the relationship between these conditions; however, the results are not consistent (4,5). Most studies

\footnotetext{
^ ORCID: 0000-0001-7795-8792.
} 
indicate that obesity can lead to emotional problems (6), especially anxiety and depression (7), and only a few suggest that obesity and mood disorders are not correlated (8). These discrepant findings might be attributed to the differences in the methods used for assessment (9) or the heterogeneity in the samples (10-12).

The possible role of central modulation in the development and recurrence of obesity is supported by a range of evidence. Recent research on obesity has gradually shifted in focus towards elucidating central modulating mechanisms to find more effective ways of preventing the occurrence of obesity $(13,14)$. The hypothalamus is widely understood to be one of the most important regions of the brain for modulating food intake and energy homeostasis. Moreover, changes in the hypothalamus influence lipolytic metabolism and thermogenesis (15). Central inflammation (mainly hypothalamic inflammation), which may be a result of an energy regulation imbalance, could be an initiating factor for obesity (16). A study of rodents on a high-fat diet (HFD) reported that food intake disorders and weight gain were associated with an increase in hypothalamic inflammation (17). HFD induced hypothalamic inflammation, which occurs prior to weight gain, and peripheral inflammation have also been suggested to be triggers of diet-induced metabolic abnormalities (18). Hypothalamic inflammation can not only cause energy imbalance, but can also aggravate insulin and leptin resistance, which in turn leads to the accumulation of fat in peripheral tissues and results in the occurrence and development of obesity (19). Once the hypothalamus has an inflammatory response, a series of metabolic abnormalities (insulin resistance, leptin resistance, etc.) and organ dysfunction (obesity, type 2 diabetes, fatty liver, and cardiovascular problems) can occur.

However, research on hypothalamic inflammation has mainly focused on animal experiments, and there are very few reports of human studies. Furthermore, these studies examined the association between hypothalamic inflammation and body mass index (BMI), but they did not investigate the association between hypothalamic inflammation and anxiety and depressive disorders. In this study, the correlation between hypothalamic inflammation and anxiety/depressive disorders in young patients with obesity was explored using a cross-sectional study design and quantitative imaging techniques. We present the following article in accordance with the STROBE reporting checklist (available at https://dx.doi.org/10.21037/apm-211480).

\section{Methods}

\section{Participants and procedures}

The study was conducted in accordance with the Declaration of Helsinki (as revised in 2013). The study was approved by the Institutional Ethics Committees of the Changzhou No. 2 People's Hospital Affiliated to Nanjing Medical University (no. 2020KY204-01). Informed consent was obtained from all participants. All participants underwent magnetic resonance imaging (MRI) and a clinical assessment based on the Self-Rating Depression Scale (SDS) and the Self-Rating Anxiety Scale (SAS) within a week of admission.

The inclusion criteria were as follows: (I) aged between 18 and 45 years, (II) at least 4 years of school education, (III) informed consent provided by either the patient or an authorized relative. Individuals with a BMI of $18.5-25 \mathrm{~kg} / \mathrm{m}^{2}$ were assigned to the control group, while individuals with a $\mathrm{BMI} \geq 30 \mathrm{~kg} / \mathrm{m}^{2}$ were assigned to the obesity group.

Exclusion criteria for all participants were as follows: (I) diagnosed with a neurological or psychological disease, (II) procalcitonin level $\geq 0.05 \mathrm{ng} / \mathrm{mL}$, (III) contraindications or inability to undergo an MRI scan, or (IV) severe hearing or vision impairment resulting in a failure to undergo assessment.

All criteria were determined by 2 experts from the Neurology Department and Psychology Department of Changzhou No. 2 People's Hospital. A professional radiologist who was blinded to the participants analyzed the images and excluded ineligible cases.

\section{Clinical assessments}

Demographic information including age, gender, height, weight, education, and renal function were collected. In addition, blood lipid, procalcitonin, fasting blood glucose, and glycosylated hemoglobin levels were collected.

The SDS and SAS have both been validated for use in adults, and both comprise 20 items. Each item assesses the frequency of symptoms as follows: $1=$ "not at all," $2=$ "sometimes," $3=$ "often," and 4= "most of the time"; the scores are reverse coded for the 5th, 9th, 13th, 17th, and 19th items. The original SDS/SAS score is the sum of individual item scores, while the standardized SDS/SAS score is the original score multiplied by 1.25 . According to the criteria used in China, individuals with a standardized SDS/SAS score $\geq 50$ are classified as having depression/ anxiety (20). 


\section{Imaging data}

Imaging data were acquired using a 3.0 Tesla MRI system (GE Discovery MR750W, GE Healthcare, Chicago, IL, USA) with an 8-channel phased-array head coil at the Radiology Department of Changzhou No. 2 People's Hospital. Participants were positioned in the supine position with their head fixed and eyes closed during the scan. T2-weighted echo planar imaging was used with the following parameters: repetition time $=4,000 \mathrm{~ms}$; echo time $=90 \mathrm{~ms}$; turn-over angle $=90^{\circ}$; slice thickness $=3 \mathrm{~mm}$; voxel size $=3.125 \times 3.15 ;$ moment matrix $=64 \times 64$; interval $=0.6 \mathrm{~mm}$; axial position, 42 layers; visual field $=240 \mathrm{~mm} \times 240 \mathrm{~mm}$; number of repeats $=160$. All scans were performed by the same radiologist.

\section{Image preprocessing and gray intensity average acquisition}

ITK-SNAP, an imaging analysis software developed by the University of Pennsylvania (USA), was used for image preprocessing and obtaining gray intensity averages. The process involved the following: (I) converting the imaging data to the DCM format, (II) importing the data into the ITK-SNAP software, (III) parameter adjustment, (IV) selecting bilateral circular regions of interest (the bilateral and most caudate brain region between the anterior optic chiasm and the posterior mammillary body; area $=10 \mathrm{~mm}^{2}$ ) and the reference region (the bilateral amygdala, which could be seen in the superior medial temporal lobe of the same section; area $\left.=10 \mathrm{~mm}^{2}\right)(21-24),(\mathrm{V})$ deriving the average gray intensity, and (VI) calculating the ratio of the hypothalamus/amygdala (H/A) .

\section{Image quality control method (24)}

Imaging data of all participants were independently analyzed by 2 trained neurologists. We assessed the reliability of the raters by evaluating variability (absolute difference between 2 raters' measurements divided by the mean of the corresponding measurements) and intragroup correlation coefficient (individual variation divided by total variation), where $<0.5$ was poor, $0.5-$ 0.75 was moderate, $>0.75-0.9$ was good, and $>0.9$ was excellent reliability. We found low internal variation (2.4\%), indicating a low measurement error, and an intragroup correlation coefficient of 0.76 , indicating considerable consistency.

\section{Statistical analyses}

All statistical analyses were conducted using SPSS version
19.0 (IBM Corp., Armonk, NY, USA), and measurement data are expressed as the mean \pm standard deviation. Normality of the data was evaluated using the KolmogorovSmirnov normality test. Differences between the 2 groups in terms of all demographic and clinical variables were assessed using a $t$ test; when parametric assumptions were not met, the Wilcoxon signed-rank test was utilized. The $\chi^{2}$ test was used to compare differences in terms of gender, education, and standardized SDS and SAS scores. Spearman correlation coefficients were used to assess associations between nonbivariate normally distributed data. A P value $<0.05$ indicated that the difference was statistically significant.

\section{Results}

\section{Participant characteristics}

Individuals admitted to the Gastrointestinal Surgery Department of Changzhou No. 2 People's Hospital between January 2019 and June 2020 were considered for enrollment in this study. The sample included 66 individuals with obesity (BMI $40.41 \pm 7.99 \mathrm{~kg} / \mathrm{m}$ ) and 44 controls (BMI $\left.22.94 \pm 1.77 \mathrm{~kg} / \mathrm{m}^{2}\right)$. There were no significant differences in terms of age, gender, or education level between the groups $(\mathrm{P}>0.05)$. Fasting blood glucose, glycated hemoglobin, lowdensity lipoprotein cholesterol, triglyceride, and uric acid levels were much lower in the control group $(\mathrm{P}<0.005)$, while high-density lipoprotein cholesterol and urea nitrogen levels were higher in the control group $(\mathrm{P}<0.05$; Table 1).

\section{Anxiety and depression scores}

In patients with obesity, the incidence of depression was higher than that in the control group $(\mathrm{P}<0.05)$, while the incidence of anxiety did not differ significantly between the 2 groups $(\mathrm{P}>0.05$; Table 2).

\section{H/A signal intensity (SI) ratio}

T2-weighted MRI images can reflect changes in brain tissue signals, and quantitative techniques can detect even more subtle ones, such as an increase in astrocyte number or microglial accumulation (25). The H/A SI ratio can be used as a surrogate marker for hypothalamic inflammation (22). The H/A SI ratio of the study group was significantly higher on the bilateral side compared with the control group $(\mathrm{P} \leq 0.001$; Table 3). In addition, we observed the $\mathrm{H} /$ 
Table 1 Participant characteristics

\begin{tabular}{|c|c|c|c|c|}
\hline Variable & Control group $(n=44)$ & Obese group $(n=66)$ & $t$ or $\chi^{2}$ & $P$ \\
\hline Gender (\%) & & & 0.03 & $>0.05$ \\
\hline Male & $18(40.91)$ & 26 (39.39) & & \\
\hline Female & $26(59.09)$ & $40(60.61)$ & & \\
\hline Primary school and below & $10(22.73)$ & $9(13.64)$ & & \\
\hline Secondary school & $9(20.45)$ & $18(27.27)$ & & \\
\hline Undergraduate and above & $25(56.82)$ & 39 (59.09) & & \\
\hline $\mathrm{BMI}\left(\mathrm{kg} / \mathrm{m}^{2}\right), \mathrm{M}(\mathrm{P} 25, \mathrm{P} 75)$ & $23.50(22.09,24.24)$ & $38.78(34.83,43.98)$ & -8.86 & $<0.001$ \\
\hline LDL-C (mmol/L) $(\bar{x} \pm \mathrm{s})$ & $2.56 \pm 0.75$ & $3.02 \pm 0.81$ & -2.99 & 0.003 \\
\hline HDL-C (mmol/L) M (P25, P75) & $1.20(0.96,1.35)$ & $1.02(0.90,1.17)$ & 2.83 & 0.005 \\
\hline TG (mmol/L), M (P25, P75) & $1.41(0.90,2.20)$ & $1.85(1.30,2.40)$ & -2.50 & 0.013 \\
\hline TC (mmol/L), M (P25, P75) & $4.48(4.02,5.21)$ & $4.68(4.22,5.48)$ & -1.14 & 0.253 \\
\hline UA (mmol/L), M (P25, P75) & $403(313.00,485.63)$ & $28,735(218.5,34,800)$ & -4.34 & $<0.001$ \\
\hline Scr (mmol/L), M (P25, P75) & $59.50(48.50,75.23)$ & $54.50(45.40,63.85)$ & 1.86 & 0.063 \\
\hline BUN (mmol/L), M (P25, P75) & $4.35(3.68,5.70)$ & $3.85(3.18,4.70)$ & 2.19 & 0.028 \\
\hline
\end{tabular}

Table 2 Prevalence of anxiety and depressive disorders

\begin{tabular}{|c|c|c|c|c|}
\hline Variable & Control group $(n=44)$ & Obese group $(n=66)$ & $\chi^{2}$ & $P$ \\
\hline Depression, N (\%) & $18(40.91)$ & $41(62.12)$ & -4.78 & $<0.05$ \\
\hline
\end{tabular}

Table 3 H/A signal intensity ratio

\begin{tabular}{lccr}
\hline Variable & Control group $(n=44)$ & Obese group $(n=66)$ & $t$ \\
\hline Right H/A ratio, M (P25, P75) & $0.99(0.95,1.04)$ & $1.08(1.01,1,15)$ & -3.96 \\
Left H/A ratio, M (P25, P75) & $1.19(0.95,1.07)$ & $1.20(1.06,1.30)$ & -4.82 \\
\hline
\end{tabular}

$\mathrm{H} / \mathrm{A}$, hypothalamus/amygdala.

A SI ratio of 11 patients with obesity before surgery and 1 year after surgery. We found that the ratios in both the left and right sides 1 year after surgery were significantly reduced compared with the corresponding side before surgery $(\mathrm{P}<0.05$; Table 4$)$.
Correlations between H/A SI ratio, BMI, and anxiety and depression scores in the obesity group

The bilateral T2-weighted image H/A SI ratio was not significantly correlated with either BMI (right: $r=-0.145$, $\mathrm{P}=0.721$; left: $\mathrm{r}=0.102, \mathrm{P}=0.415$ ) or SAS score (right: $\mathrm{r}=$ 
Table 4 H/A signal intensity ratio before and after surgery

\begin{tabular}{|c|c|c|c|c|}
\hline Variable & Before surgery $(n=11)$ & After surgery $(n=11)$ & $t$ & $P$ \\
\hline Left H/A ratio, $(\bar{x} \pm s)$ & $1.18 \pm 0.16$ & $1.04 \pm 0.14$ & 2.30 & $<0.05$ \\
\hline
\end{tabular}

$\mathrm{H} / \mathrm{A}$, hypothalamus/amygdala.
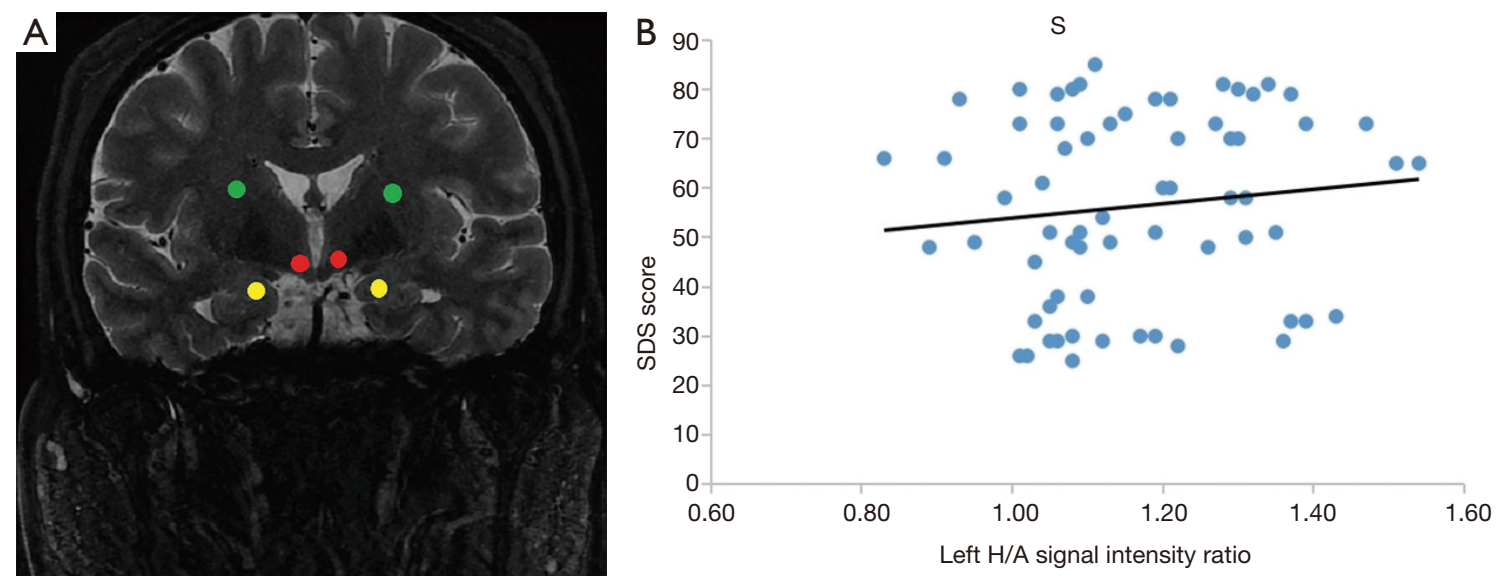

Figure 1 ROIs in coronal T2-weighted images and SDS score. (A) Representative coronal T2-weighted MRI scan through the hypothalamus. The zoomed image on the right shows the placement of the right and left ROIs. The red-, yellow-, and green-stained areas represent the regions of interest in the hypothalamus, amygdala, and putamen. (B) Correlations between the left hypothalamus/amygdala signal ratio and SDS score. SDS, Self-Rating Depression Scale; MRI, magnetic resonance imaging; ROIs, regions of interest.

$-0.118, \mathrm{P}=0.444$; left: $\mathrm{r}=0.295, \mathrm{P}=0.052$ ) in the obesity group; right $\mathrm{T} 2$-weighted image $\mathrm{H} / \mathrm{A}$ SI ratio was not significantly associated with SDS score $(\mathrm{r}=-0.031, \mathrm{P}=0.843)$, but left T2-weighted image H/A SI ratio was significantly associated with SDS score $(\mathrm{r}=-0.353, \mathrm{P}=0.019$; Figure 1$)$.

\section{Discussion}

Obesity, along with anxiety and depressive disorders, is a major public health problem. These conditions independently affect disability and mortality rates in patients and incur a considerable socioeconomic burden; moreover, the results of several epidemiological studies, clinical trials, and recent meta-analyses support the link between mood disorders and obesity $(7,9,10,26)$. Depression and anxiety are particularly prominent among mood disorders, not only because of their high prevalence but also because of their significant association with obesity $(9,27)$. In this study, the incidence of depression in the obesity group was found to be higher than that in the control group, which is in line with previous studies $(7,9,10,27,28)$. Studies have also found that the effects of obesity and depression work bidirectionally (26-29) and that individuals who are obese have an $18-40 \%$ increased risk of depression $(28,29)$. Obesity had similar odds ratios (ORs) for depression (OR 1.21-5.8) and vice versa (OR: 1.18-3.76) (26). People with depression may gain significant weight due to clinical symptoms and/or medication-induced irregular eating patterns and sedentary lifestyles. The tendency to overeat due to negative emotions, known as emotional eating, is also closely associated with weight gain (30). By contrast, the link between anxiety and obesity is not particularly strong. The pooled OR of an association between obesity and anxiety was 1.4 [confidence interval (CI): 1.23-1.57] (31). Similarly, the difference in the incidence of anxiety between the obese group and the control group in this study was not found to be statistically significant.

Hypothalamic inflammation is the process in which hypothalamic neuronal or nonneuronal cells, mainly microglia and astrocytes, directly or indirectly participate in the activation of proinflammatory signals in response to a variety of triggers (32). Animal studies have shown that 
diets high in fat or saturated fat (33) activate microglia in the hypothalamus in the short term and that this precedes weight gain (14). Astrocytes, which are also glial cells, are abundant in the central nervous system and support neurological function in a variety of ways (34). Longterm consumption of a HFD increases the number of glial fibrillary acidic protein-positive astrocytes in a process called reactive astrocyte proliferation; these reactive astrocytes may participate in the development of obesity by altering the release or uptake of neurotransmitters, such as gamma-aminobutyric acid, that promote increased food intake (35). This suggests that the hypothalamus is one of the most sensitive organs to HFD-induced inflammation or immune activation and that hypothalamic inflammation may not only be a consequence of established obesity but an important contributor to the development of obesity (14). Although hypothalamic inflammation has been investigated in several independent animal studies, due to technical and methodological limitations, data on human hypothalamic inflammation are still scarce. Thaler et al. (21) were the first to use H/A SI ratio in the MRI T2 coronal plane to assess hypothalamic inflammation. A retrospective study also found that the left H/A SI ratio in the obesity group was significantly higher than that in the control group (21). Similar results were confirmed 3 years later (22); specifically, an association was found between the MRI data and the histopathology of astrocytosis both from vivo and the postmortem subjects, thus proving that quantitative MRI techniques can detect hypothalamus inflammation and gliosis (23). Using a similar method, we assessed the H/ A SI ratio in the obesity group and the control group and found that the H/A ratios on both the left and the right sides were significantly higher than those in the control group, which is consistent with previous studies $(22,23)$. This suggests that glial hyperplasia is bilateral. In addition, one of the most effective treatments, bariatric surgery can alleviate hypothalamic inflammation (36), which was also confirmed by our research, also demonstrating the role of hypothalamic inflammation in the process of obesity.

We also analyzed the T2-MRI data to assess whether $\mathrm{BMI}$ and SAS and SDS scores were associated with the H/A SI ratio. Regarding the correlations with BMI in this study, we were not able to replicate the results of previous studies (21-23); this may be due to our small sample size or other technical reasons (24). The hypothalamus is a very small brain region, and our T2-MRI sequence was protocoled to cover the whole brain with $3 \mathrm{~mm}$ thick slices. These comparatively thick slices might have caused region-of- interest mispositioning. In regard to the correlation with anxiety scores, our results are similar to those of previous studies (9-11) although the left H/A signal ratio was not significantly correlated with anxiety scores. Our results showed that the left H/A signal ratio was correlated with the depression score, but there was no significant correlation with the right-side H/A signal ratio. An association between the left hypothalamus and depression was noted by Schindler et al. (37), who found the left hypothalamus volume to be increased in patients with major depression but no such correlation with the right hypothalamus. Previous studies $(21-23,38)$ have confirmed the association between H/A SI ratio and BMI. We speculate that there is a link between obesity and depression, and inflammation of the left hypothalamus may be one of the links between the two. Further information in this regard could provide valuable insights that would help to better manage and treat these conditions.

Our study has certain limitations. First, it was designed to be a cross-sectional study, which can only be used to analyze correlations, not causation. Second, the sample size of our study was relatively small, and future studies with larger sample sizes will be needed to verify the results. Finally, quantitative study of hypothalamic inflammation with enhanced image quality control methods to improve the consistency of the H/A SI ratio will be required to obtain more accurate data.

\section{Conclusions}

Patients with obesity were found to have a higher incidence of depression. Our results also indicated that inflammation of the left hypothalamus may be one of the links between obesity and depression.

\section{Acknowledgments}

We would like to recognize Dr. Jun Qian and his team, who had performed surgical procedures independently. We would also like to thank Dr. Xinhua Ye for providing followup data and MRI scans of patients after surgery.

Funding: This study was supported by the National Natural Science Foundation of China (no. 81671284).

\section{Footnote}

Reporting Checklist: The authors have completed the STROBE reporting checklist. Available at https://dx.doi. 
org/10.21037/apm-21-1480

Data Sharing Statement: Available at https://dx.doi. org/10.21037/apm-21-1480

Conflicts of Interest: All authors have completed the ICMJE uniform disclosure form (available at https://dx.doi. org/10.21037/apm-21-1480). The authors have no conflicts of interest to declare.

Ethical Statement: The authors are accountable for all aspects of the work in ensuring that questions related to the accuracy or integrity of any part of the work are appropriately investigated and resolved. The study was conducted in accordance with the Declaration of Helsinki (as revised in 2013). The study was approved by the Institutional Ethics Committees of the Changzhou No. 2 People's Hospital Affiliated to Nanjing Medical University (no. 2020KY204-01), and informed consent was obtained from all participants.

Open Access Statement: This is an Open Access article distributed in accordance with the Creative Commons Attribution-NonCommercial-NoDerivs 4.0 International License (CC BY-NC-ND 4.0), which permits the noncommercial replication and distribution of the article with the strict proviso that no changes or edits are made and the original work is properly cited (including links to both the formal publication through the relevant DOI and the license). See: https://creativecommons.org/licenses/by-nc-nd/4.0/.

\section{References}

1. Ogden CL, Carroll MD, Kit BK, et al. Prevalence of childhood and adult obesity in the United States, 20112012. JAMA 2014;311:806-14.

2. Sanyaolu A, Okorie C, Qi X, et al. Childhood and Adolescent Obesity in the United States: A Public Health Concern. Glob Pediatr Health 2019;6:2333794X19891305.

3. Guo Y, Yin X, Wu H, et al. Trends in Overweight and Obesity Among Children and Adolescents in China from 1991 to 2015: A Meta-Analysis. Int J Environ Res Public Health 2019;16:4656.

4. Ribeiro O, Carmo I, Paiva T, et al. Neuropsychological Profile, Cognitive Reserve and Emotional Distress in a Portuguese Sample of Severely Obese Patients. Acta Med Port 2020;33:38-48.
5. Hou Q, Guan Y, Yu W, et al. Associations between obesity and cognitive impairment in the Chinese elderly: an observational study. Clin Interv Aging 2019;14:367-73.

6. Casagrande M, Boncompagni I, Forte G, et al. Emotion and overeating behavior: effects of alexithymia and emotional regulation on overweight and obesity. Eat Weight Disord 2020;25:1333-45.

7. Rajan TM, Menon V. Psychiatric disorders and obesity: A review of association studies. J Postgrad Med 2017;63:182-90.

8. Wild B, Herzog W, Lechner S, et al. Gender specific temporal and cross-sectional associations between BMI-class and symptoms of depression in the elderly. J Psychosom Res 2012;72:376-82.

9. Wang S, Sun Q, Zhai L, et al. The Prevalence of Depression and Anxiety Symptoms among Overweight/ Obese and Non-Overweight/Non-Obese Children/ Adolescents in China: A Systematic Review and MetaAnalysis. Int J Environ Res Public Health 2019;16:340.

10. Moradi M, Mozaffari H, Askari M, et al. Association between overweight/obesity with depression, anxiety, low self-esteem, and body dissatisfaction in children and adolescents: a systematic review and meta-analysis of observational studies. Crit Rev Food Sci Nutr 2020. [Epub ahead of print]. doi: 10.1080/10408398.2020.1823813.

11. Roberts RE, Hao DT. Obesity has few effects on future psychosocial functioning of adolescents. Eat Behav 2013;14:128-36.

12. Rivenes AC, Harvey SB, Mykletun A. The relationship between abdominal fat, obesity, and common mental disorders: results from the HUNT study. J Psychosom Res 2009;66:269-75.

13. Seong J, Kang JY, Sun JS, et al. Hypothalamic inflammation and obesity: a mechanistic review. Arch Pharm Res 2019;42:383-92.

14. Jais A, Brüning JC. Hypothalamic inflammation in obesity and metabolic disease. J Clin Invest 2017;127:24-32.

15. Trayhurn P. Origins and early development of the concept that brown adipose tissue thermogenesis is linked to energy balance and obesity. Biochimie 2017;134:62-70.

16. Saltiel AR, Olefsky JM. Inflammatory mechanisms linking obesity and metabolic disease. J Clin Invest 2017;127:1-4.

17. O'Rahilly S, Farooqi IS, Yeo GS, et al. Minireview: human obesity-lessons from monogenic disorders. Endocrinology 2003; 144:3757-64.

18. Kim JD, Yoon NA, Jin S, et al. Microglial UCP2 Mediates Inflammation and Obesity Induced by High-Fat Feeding. Cell Metab 2019;30:952-62.e5.

19. Benomar Y, Taouis M. Molecular Mechanisms Underlying 
Obesity-Induced Hypothalamic Inflammation and Insulin Resistance: Pivotal Role of Resistin/TLR4 Pathways. Front Endocrinol (Lausanne) 2019;10:140.

20. Yue T, Li Q, Wang R, et al. Comparison of Hospital Anxiety and Depression Scale (HADS) and Zung SelfRating Anxiety/Depression Scale (SAS/SDS) in Evaluating Anxiety and Depression in Patients with Psoriatic Arthritis. Dermatology 2020;236:170-8.

21. Thaler JP, Yi CX, Schur EA, et al. Obesity is associated with hypothalamic injury in rodents and humans. J Clin Invest 2012;122:153-62.

22. Schur EA, Melhorn SJ, Oh SK, et al. Radiologic evidence that hypothalamic gliosis is associated with obesity and insulin resistance in humans. Obesity (Silver Spring) 2015;23:2142-8.

23. Kreutzer C, Peters S, Schulte DM, et al. Hypothalamic Inflammation in Human Obesity Is Mediated by Environmental and Genetic Factors. Diabetes 2017;66:2407-15.

24. Rebelos E, Hirvonen J, Bucci M, et al. Brain free fatty acid uptake is elevated in morbid obesity, and is irreversible 6months after bariatric surgery: A positron emission tomography study. Diabetes Obes Metab 2020;22:1074-82.

25. Lee D, Thaler JP, Berkseth KE, et al. Longer T(2) relaxation time is a marker of hypothalamic gliosis in mice with diet-induced obesity. Am J Physiol Endocrinol Metab 2013;304:E1245-50.

26. Dionysopoulou S, Charmandari E, Bargiota A, et al. The Role of Hypothalamic Inflammation in Diet-Induced Obesity and Its Association with Cognitive and Mood Disorders. Nutrients 2021;13:498.

27. Husky MM, Mazure CM, Ruffault A, et al. Differential Associations Between Excess Body Weight and Psychiatric Disorders in Men and Women. J Womens Health (Larchmt) 2018;27:183-90.

28. Du FM, Kuang HY, Duan BH, et al. Effects of thyroid hormone and depression on common components of central obesity. J Int Med Res 2019;47:3040-9.

Cite this article as: Dong GZ, Yang HJ, Jiao YW, Zhang QY, Ma Y, Zhu SM, Zhang LH, Zhang M, Yao T, Cao Y, Tang LM. Using quantitative imaging to determine the correlation between hypothalamic inflammation and anxiety and depression in young patients with obesity. Ann Palliat Med 2021;10(7):80438050. doi: 10.21037/apm-21-1480
29. Mannan M, Mamun A, Doi S, et al. Is there a bidirectional relationship between depression and obesity among adult men and women? Systematic review and biasadjusted meta analysis. Asian J Psychiatr 2016;21:51-66.

30. Frayn M, Knäuper B. Emotional eating and weight in adults: a review. Curr Psychol 2018;37:924-33.

31. Gariepy G, Nitka D, Schmitz N. The association between obesity and anxiety disorders in the population: a systematic review and meta-analysis. Int J Obes (Lond) 2010;34:407-19.

32. Cai D, Khor S. "Hypothalamic Microinflammation" Paradigm in Aging and Metabolic Diseases. Cell Metab 2019;30:19-35.

33. Valdearcos M, Robblee MM, Benjamin DI, et al. Microglia dictate the impact of saturated fat consumption on hypothalamic inflammation and neuronal function. Cell Rep 2014;9:2124-38.

34. Rahman MH, Bhusal A, Kim JH, et al. Astrocytic pyruvate dehydrogenase kinase-2 is involved in hypothalamic inflammation in mouse models of diabetes. Nat Commun 2020;11:5906.

35. Zhang Y, Reichel JM, Han C, et al. Astrocytic Process Plasticity and IKK $\beta / \mathrm{NF}-\kappa \mathrm{B}$ in Central Control of Blood Glucose, Blood Pressure, and Body Weight. Cell Metab 2017;25:1091-1102.e4.

36. Herrick MK, Favela KM, Simerly RB, et al. Attenuation of diet-induced hypothalamic inflammation following bariatric surgery in female mice. Mol Med 2018;24:56.

37. Schindler S, Schmidt L, Stroske M, et al. Hypothalamus enlargement in mood disorders. Acta Psychiatr Scand 2019;139:56-67.

38. Zou B, Miao C, Chen J. Depression and Perceived Stress, but Not Anxiety, are Associated with Elevated Inflammation in an Obese Adult Population. Risk Manag Healthc Policy 2020;13:1489-97.

(English Language Editors: B. Meiser and J. Gray) 